\title{
Vibration monitoring of rotor bearing system using MFS-lite
}

\author{
Abdul Khaliq Ansari', Zain Ahmad Khan', Ahmad Ali Khan ${ }^{3}$ \\ Department of Mechanical Engineering, Zakir Husain College of Engineering and Technology, \\ Aligarh Muslim University, Aligarh, India \\ ${ }^{1}$ Corresponding author \\ E-mail: 19abdulkhaliq@zhcet.ac.in, ${ }^{2} z$ ainakhan1000@gmail.com, ${ }^{3}$ khanaali@rediffmail.com \\ Received 11 November 2018; accepted 20 November 2018 \\ DOI https://doi.org/10.21595/vp.2018.20364 \\ Check for updates \\ Copyright (C) 2018 Abdul Khaliq Ansari, et al. This is an open access article distributed under the Creative Commons Attribution License, \\ which permits unrestricted use, distribution, and reproduction in any medium, provided the original work is properly cited.
}

\begin{abstract}
Vibrations in rotating machinery are commonly the result of mechanical faults including mass unbalance, coupling misalignment, mechanical looseness etc. Rotating machinery with overhung rotors is very common in industries. Unbalanced rotor and misaligned shafts usually cause excessive machine vibration, generates large forces on bearings and thus reduces the machine life span and may lead to property loss and even loss of human life. Two-plane balancing of overhung rotors is one of the most challenging problem that maintenance engineers may encounter. As a prerequisite, successful diagnosis of unbalanced overhung rotor system must be performed. The vibration signatures of unbalanced overhung rotors with unknown initial conditions are different from those of the systems with centre hung rotors that has been studied in the present work. Experiments were carried out on a Machinery Fault Simulator-Lite (MFS-Lite) for both balanced and unbalanced rotor systems. The data were analysed using the Balance Quest software and efforts were focused on identifying the system characteristic signatures. Further, the results were verified through analytical methods and the results were found to be satisfactory.
\end{abstract}

Keywords: two plane rotor balancing, balance quest, MFS lite, influence coefficient method.

\section{Nomenclature}

$A_{N} \quad$ Initial vibration vector at near bearing

$A_{F} \quad$ Initial vibration vector at far bearing

$H_{N N} \quad$ Influence vector at near bearing to weight at near plane

$H_{N F} \quad$ Influence vector at near bearing to weight at far plane

$H_{F N} \quad$ Influence vector at far bearing to weight at near plane

$H_{F F} \quad$ Influence vector at far bearing to weight at far plane

$C W_{N}$ Mass correction vector at near plane

$C W_{F}$ Mass correction vector at far plane

$B_{N N} \quad$ Vibration vector at near bearing with weight at near plane

$B_{F N} \quad$ Vibration vector at far bearing with weight at near plane

$W_{N} \quad$ Calibration weight vector at near plane

$B_{N F} \quad$ Vibration vector at near bearing with weight at far plane

$B_{F F} \quad$ Vibration vector at far bearing with weight at far plane

$W_{F} \quad$ Calibration weight vector at far plane

\section{Introduction}

A small amount of unbalance weight in rotating system may have devastating effects as the system is operating with high speed or running near the critical speed. Therefore, extreme care must be taken in balancing high-speed rotating system to avoid any potential damages. Overhung rotors are commonly used in fluid turbo-machinery, such as pumps, propellers and fans. Balancing is the process of attempting to improve the mass distribution of a body so that it rotates in its bearings without unbalanced centrifugal forces. It is important to recognize that most balancing 
operations are performed below the first critical speed. To balance above the first critical requires different techniques and may be supported by another type of software. Balancing is the procedure of measuring vibration and adding or removing weight to adjust the mass distribution. The final goal is to reduce vibration level. Mass balancing is only one remedial action for excessive vibrations from machines.

Several review papers in the area of rotor balancing have been published over the past years that includes Parkinson [1] who reviewed in his book the state of the art on unbalance response and balancing of rigid and flexible rotor, he has also included a description of experimental work and a comparison between the different balancing methods. Van de Vegte [2] proposed the idea of balancing without interrupting operation, but that work concentrated on theoretical problems associated with the possibility of closed loop automatic control. Srinivasan [3] has done comprehensive experimental studies in the rotating machinery for the faults like misalignment (parallel misalignment from $0.025 \mathrm{~mm}$ to $0.65 \mathrm{~mm}$, angular misalignment from 0.02 degree to 0.6 degree), unbalance (105.06 gm-cm to $491.98 \mathrm{gm}-\mathrm{cm})$, mechanical looseness, rotor rub, bearing clearance (from 0.02 to $0.08 \mathrm{~mm}$ ) and crack (transverse cracks of width $0.75 \mathrm{~mm}$ and depth ranging from 1.02 to $4.02 \mathrm{~mm}$ at the mid span of the shaft). Pandey and Nakra [4] provide a valuable information regarding symptoms of machinery failure which in practice may avoid costly break downs. The fault like parallel misalignment, angular misalignment, combined parallel and angular misalignment and unbalance have been simulated. In all cases amplitudes are higher at NDE (Non-Drive End) and lesser at DE (Drive End). The amplitude is higher in axial direction in both the ends and increased whenever misalignment increased. Vyas and Satish [5] have carried out experimental studies to generate data and discussed the development of neural network simulator for prediction of faults like mass unbalance, bearing cap looseness, play in spider coupling and rotor with both mass unbalance and misalignment and health machine network. The test data were generated on a laboratory rotor rig which consist of a $10 \mathrm{~mm}$ diameter shaft carrying a centrally located steel disk ( $0.5 \mathrm{~kg}$ mass) and supported in identical rolling element bearing (6200 SKF ball bearing) at the two bearing and driven by 50-Watt, 230 Volt AC/DC electric motor through a flexible spider coupling. Fault simulated were: Mass unbalance, Loose Bearing cap \& Misalignment and both mass unbalance \& misalignment.

\section{Experimental setup for balancing of rotor}

The experimental setup used in this study is shown in Fig. 1. This apparatus is driven by a 3-HP induction motor with a speed range between 20 and $3000 \mathrm{rpm}$. The shaft rotation speed can be controlled by a speed controller.

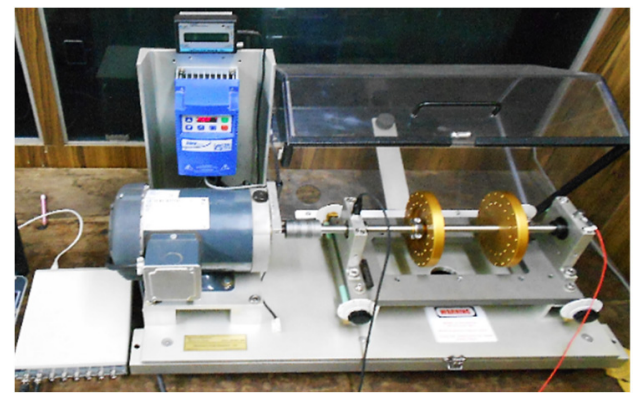

Fig. 1. Experimental setup for two plane rotor system

An optical sensor is used for shaft-speed measurement. A flexible coupling is utilized to damp out the high-frequency vibration that was generated by the motor. Two rolling-element bearings are fitted in the solid housings. Accelerometers are mounted on the housing of the tested bearing to measure the vibration signals along two directions. Based on the structure properties, the signal that was vertically measured is utilized for analysis in this study, whereas the information from 
the signal that was horizontally measured is used for verification. Data were acquired using Spectra PAD data acquisition board at $51.2 \mathrm{kHz}$ sampling rate for about 32 seconds.

Vibrations from the inboard bearing and outboard bearings were measured in vertical directions using accelerometers. Tachometer in the MFS is used to measure the exact shaft speed. The tests are taken with the Aluminium Rotor. The two conditions of rotor are considered namely; 1. Centerhung rotor and 2. Overhung rotor.

\section{Balancing using BQ software}

In two plane rotor system, the test was carried out at $15 \mathrm{~Hz}$ for centrehung loading. The Correction Weight for balancing the rotor are obtained for both the cases which are as follows:

For Centrehung loading: $C W_{F}=1.214 \angle 122.72 \mathrm{gm}, C W_{N}=10.476 \angle 205.82 \mathrm{gm}$,

For Overhung loading: $C W_{F}=6.1268 \angle 298.79 \mathrm{gm}, C W_{N}=5.3770 \angle 148.53 \mathrm{gm}$.

The sample reading obtained from BQ Software for $15 \mathrm{~Hz}$ centrehung loading are.

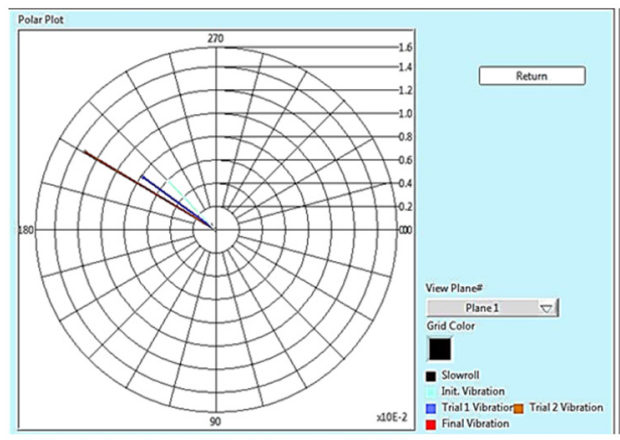

a)

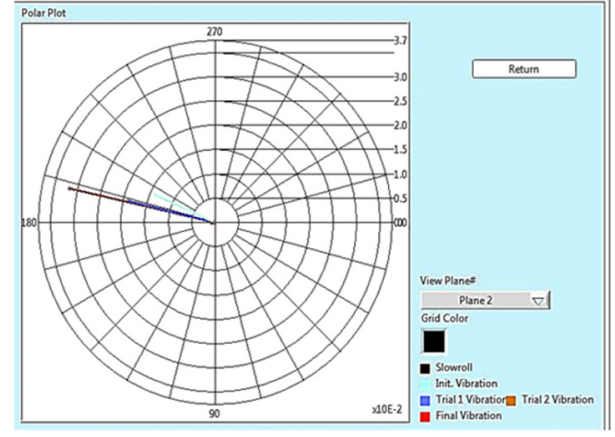

b)

\begin{tabular}{|l|l|l|l|l|l|}
\hline \#ofPlanes & 2 & & & & \\
\hline Method & Inf. Coef. & & & & \\
\hline & & & & & \\
\hline Step & Plane\# & Vib. Amplitud & Vib. Angle & Weight & Angle of \\
\hline Slowroll & 1 & 0.000000 & 0.00 & -- & -- \\
\hline Slowroll & 2 & 0.000000 & 0.00 & -- & -- \\
\hline Initial Vibration & 1 & 0.005806 & 225.34 & -- & -- \\
\hline Initial Vibration & 2 & 0.013730 & 203.51 & -- & -- \\
\hline Trial Run 1 & 1 & 0.007754 & 215.48 & 4.320000 & 40.00 \\
\hline Trial Run 1 & 2 & 0.018597 & 193.42 & -- & -- \\
\hline Trial Run 2 & 1 & 0.013104 & 210.27 & -- & -- \\
\hline Trial Run 2 & 2 & 0.031238 & 192.72 & 4.320000 & 40.00 \\
\hline Solution & 1 & - & -- & 10.476238 & 205.82 \\
\hline Solution & 2 & -- & -- & 1.214526 & 122.72 \\
\hline Final Vibration & 1 & 0.000543 & 230.08 & -- & -- \\
\hline Final Vibration & 2 & 0.000918 & 152.40 & -- & -- \\
\hline
\end{tabular}

c)

Fig. 2. Polar plot for plane 1, 2 and Tabular result for $15 \mathrm{~Hz}$

Fig. 3(a)-(d) shows the Spectrum analysis (i.e. Amplitude Vs Frequency) of two rotor system at $15 \mathrm{~Hz}(900 \mathrm{rpm})$. The amplitudes are measured in $\mathrm{mm}$ whereas the frequency in Hz. Fig. 3(a) shows the initial vibration at DE/Near End and NDE/Far End. Fig. 3(b) shows the trial vibration 1 at DE and NDE. Fig. 3(c) shows the trial vibration 2 at DE and NDE. Fig. 3(d) shows the Final vibration at DE and NDE respectively, after the removal of unbalance in the rotor.

Fig. 2 shows the polar plot of two rotor system at $15 \mathrm{~Hz}(900 \mathrm{rpm})$ for both the plane i.e. plane 1 and plane 2. Polar plot shows in initial unbalance response present in the rotor, response with trial weight of 4.32 gm placed at plane 1, response with trial weight of 4.32 gm placed at plane 2 and the final vibration after the removal of unbalance in the rotor, and also shows the complete result of balancing in tabular form in which initial unbalance in near and far plane are $5.806 \mu \mathrm{m}$ and $13.730 \mu \mathrm{m}$ respectively. After placing trial weight of $4.32 \mathrm{gm}$ in near plane (Plane 1), we got the vibration amplitude $7.754 \mu \mathrm{m}$ in near plane and $18.597 \mu \mathrm{m}$ at far plane. 
Similarly, after placing trial weight of $4.32 \mathrm{gm}$ in far plane (Plane 2), we got the vibration amplitude $13.104 \mu \mathrm{m}$ in near plane and $31.238 \mu \mathrm{m}$ at far plane.

Using the data recorded from the previous steps, BQ Software calculates the correction weight size and location i.e. $10.47 \mathrm{gm}$ at $\angle 205.82$ in plane 1 and $1.21 \mathrm{gm}$ at $\angle 122.7$ in plane 2 . By placing these weights in the desired location, we got the final vibration amplitude that is equal to $0.543 \mu \mathrm{m}$ in near plane and $0.918 \mu \mathrm{m}$ at far plane, which is almost negligible in comparison to original response as shown in Fig. 3(d).
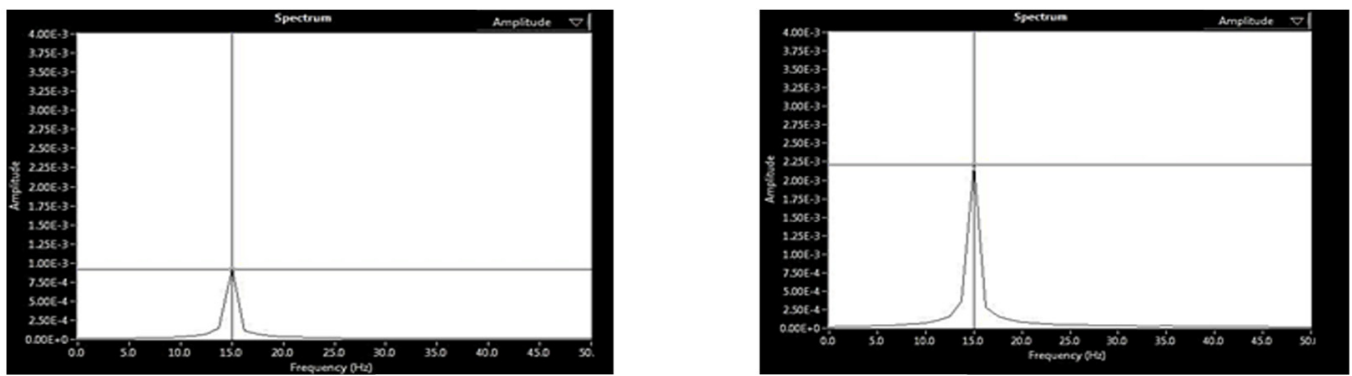

a) Initial vibration at DE and NDE

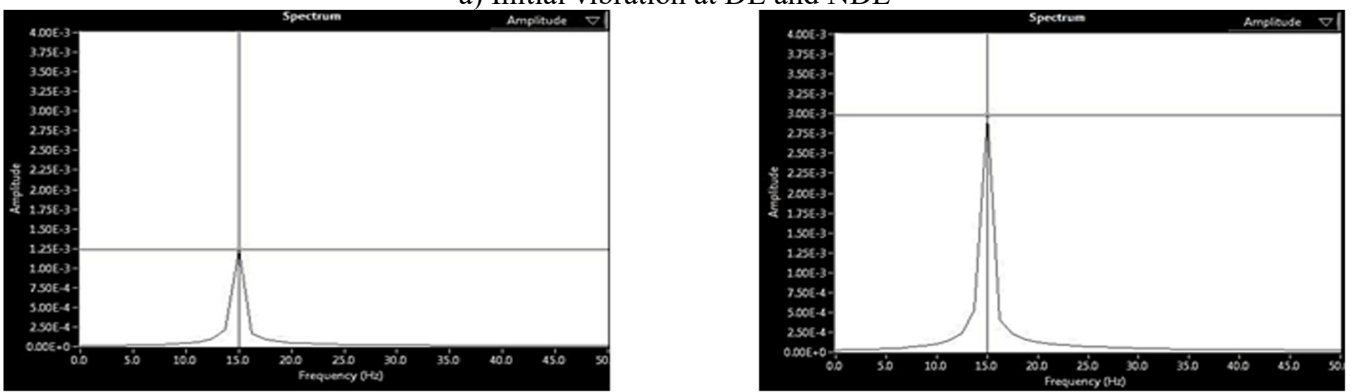

b) Trial 1 vibration at DE and NDE
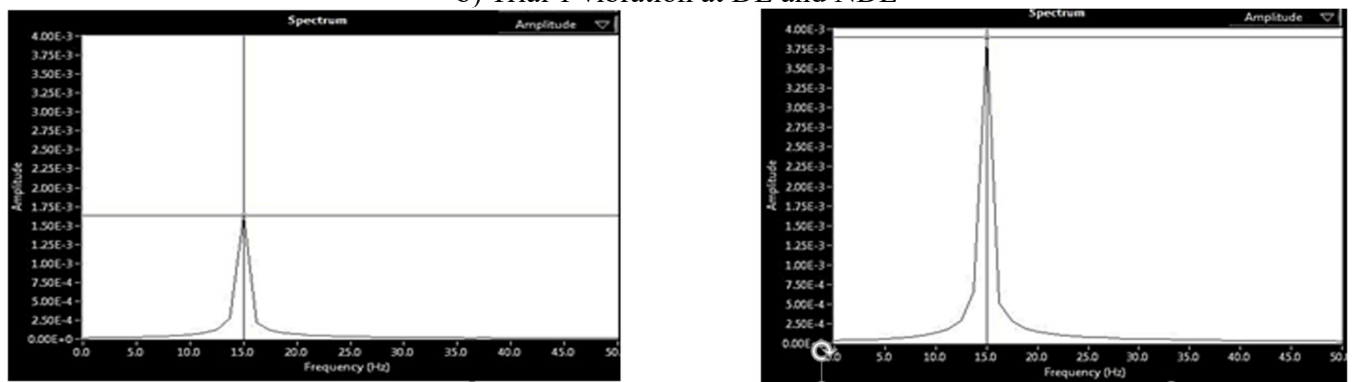

c) Trial 2 vibration at $\mathrm{DE}$ and $\mathrm{NDE}$
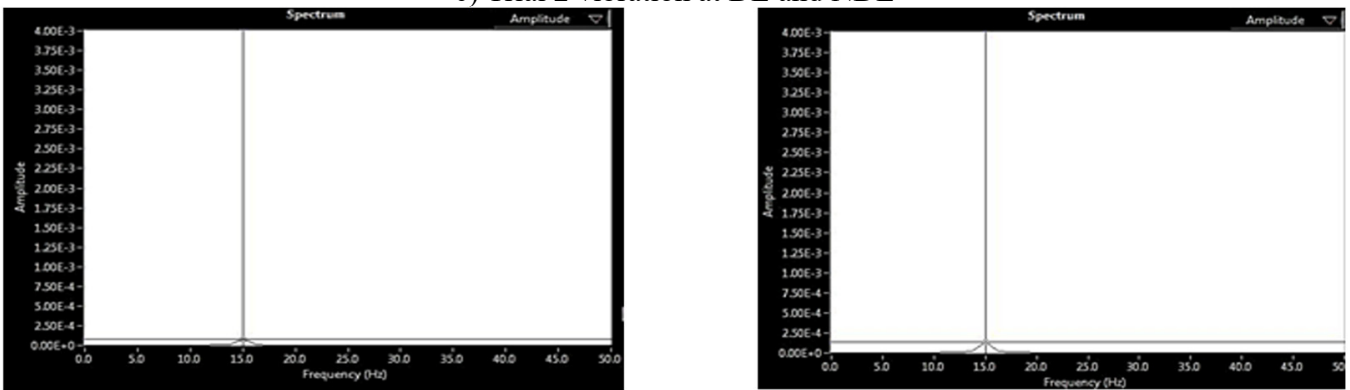

d) Final vibration at $\mathrm{DE}$ and NDE

Fig. 3. Balancing of single plane rotors at $15 \mathrm{~Hz}(900 \mathrm{rpm})$ 


\section{Balancing using influence coefficient method}

We have validated BQ results obtained above with the Analytical Method. i.e. Influence coefficient or Vector Method, where the role of phase is very important point of consideration.

Note: In BQ calculations, the phase is done as phase-lead convention and our weight location is defined as "angle in anti-rotating direction from reflective tape/key way", which is a phase lag convention. And that's why we have to convert it into phase-lead by using "360 minus weight spot" or Converting the trial weight location to phase lead by simply multiplying its angle by $(-1)$, do the calculations \& convert the correction weight back into phase lag by multiplying its angle by $(-1)[6]$.

So, it's all about which direction to count the angle, and as long as we keep the phase convention consistent in the calculation, we will get the correct answer.

The sample calculation for two plane Centerhung [7, 8] at $15 \mathrm{~Hz}$, is mention below.

The initial vibration readings obtained on a rigid-body rotor are: $A_{N}=5.806 \angle 225.34 \mu \mathrm{m}$ and $A_{F}=13.73 \angle 203.51 \mu \mathrm{m}$.

A trial balance weight of $W_{N}=4.32 \mathrm{gm}$ is placed at a relative phase angle of $40^{\circ}$. The resulting vibration at near plane and far plane due to the placement of the trial weight at near plane are: $B_{N N}=7.754 \angle 215.48 \mu \mathrm{m}$ and $B_{F N}=18.597 \angle 193.42 \mu \mathrm{m}$

The first balance trial weight is removed, and a trial weight of $W_{F}=4.32 \mathrm{gm}$ is placed at a relative phase angle of $40^{\circ}$ at far plane. The resulting vibration readings are: $B_{N F}=13.104 \angle 210.27 \mu \mathrm{m}$ and $B_{F F}=31.238 \angle 192.72 \mu \mathrm{m}$.

For calculation in phase lag, we convert all the phase lead angle by multiplying " -1 ".

$A_{N}=5.80 \angle-225.34 \mu \mathrm{m}, A_{F}=13.73 \angle-203.51 \mu \mathrm{m}, B_{N N}=7.75 \angle-215.48 \mu \mathrm{m}$,

$B_{N F}=13.10 \angle-210.27 \mu \mathrm{m}, B_{F N}=18.59 \angle-193.42 \mu \mathrm{m}, B_{F F}=31.23 \angle-192.72 \mu \mathrm{m}$.

The influence coefficients at near and far plane caused by the trial weight placed at near plane are given by:

$\overrightarrow{H_{F N}}=\frac{\overrightarrow{B_{F N}}-\overrightarrow{A_{F}}}{\vec{W}_{N}}=1.301 \angle 151.921, \overrightarrow{H_{N N}}=\frac{\overrightarrow{B_{N N}}-\overrightarrow{A_{N}}}{\vec{W}_{N}}=0.524 \angle 130.572$.

The influence coefficients at near and far plane caused by the trial weight placed at far plane are given by:

$\overrightarrow{H_{N F}}=\frac{\overrightarrow{B_{N F}}-\overrightarrow{A_{N}}}{\vec{W}_{F}}=1.770 \angle 121.114, \overrightarrow{H_{F F}}=\frac{\overrightarrow{B_{F F}}-\overrightarrow{A_{F}}}{\vec{W}_{F}}=4.152 \angle 135.519$,

Balance correction:

$C W_{N}=\frac{A_{F} H_{N F}-A_{N} H_{F F}}{H_{F F} H_{N N}-H_{N F} H_{F N}}=10.503 \angle 205.78 \mathrm{gm}$,

$C W_{F}=\frac{A_{N} H_{F N}-A_{F} H_{N N}}{H_{F F} H_{N N}-H_{N F} H_{F N}}=1.216 \angle 122.48 \mathrm{gm}$.

We have done the sample calculation for $15 \mathrm{~Hz}$ in phase lag. To check the correctness of our experimental result, that is obtained from BQ Software. We have compared these results for two planes (Centerhung) from Analytical Results which is calculated from Influence Coefficient Method and has been shown in Table. 2. Further, these results have been found to be in very good agreement with each other. 
Table 2. Comparison of correction weight for two plane balancing

\begin{tabular}{|c|c|c|c|c|}
\hline \multirow{2}{*}{ Speed (Hz) } & \multicolumn{4}{|c|}{ Correction weight (gm) } \\
\cline { 2 - 5 } & Far plane & Near plane & Influence coefficient method \\
\cline { 2 - 5 } & $1.214526 \angle 122.72$ & $10.476238 \angle 205.82$ & $1.216 \angle 122.48$ & $10.503 \angle 205.78$ \\
\hline 15 (Centerhung) & Far plane & Near plane \\
\hline 15 (Overhung) & $6.126859 \angle 298.79$ & $5.377068 \angle 148.53$ & $6.11 \angle 298.752$ & $5.365 \angle 149.54$ \\
\hline
\end{tabular}

\section{Conclusions}

The conventional diagnosis method to diagnose faults in the rolling element bearing is not applicable for a rotor bearing system when the rotor operates at low/slow speed. The Experimental Results for Single Plane and Two Plane Centerhung and Overhung rotor conditions were carried out with different operation speed, different unbalance type and different unbalance weight. This has been shown by Analytical Method i.e. (Influence Coefficient Method) in order to check the correctness of the results. The results have been found to be in very close agreement with each other, which proves the correctness and robustness of the Experiment using MFS-Lite. The present study can also be used for online condition monitoring of rotor bearing system.

Experimental results of present study clearly indicate that Rotating system with overhung rotors, the existence of unbalance is closely related to two important signatures: the $1 \mathrm{X}$ radial force/acceleration spectrum magnitude, and more significantly and strongly, related to outboard bearing (the one close to the overhung rotor) $1 \mathrm{X}$ axial acceleration spectrum magnitude.

The bearing forces, and thus the sensitivity to unbalance, is proportional to the square of speed of rotation. Therefore, when more sensitivity required, higher balancing speed is used. Proper trial weight (TW) selection is important because a weight that is too light may not provide an adequate response for calculating correction weights and their placement. A trial weight that is too large may wreck the machine. Trial weights should adhere to the 30/30 rule [9] that says change the phase by 30 degrees or the amplitude by at least 30 percent. Experimental results have confirmed some observation from published literature that the fault like parallel misalignment, angular misalignment, combined parallel and angular misalignment and unbalance, in all cases amplitudes are higher at NDE and lesser at DE [4].

\section{References}

[1] Parkinson A. G. Balancing of rotating machinery. Proceedings of the Institution of Mechanical Engineers, Part C: Journal of Mechanical Engineering Science, Vol. 205, Issue 1, 1991, p. 53-66.

[2] Van De Vegte Continuous automatic balancing of rotating systems. The Journal of Mechanical Engineering Science, Vol. 6, Issue 3, 1964, p. 264-269.

[3] Srinivasan K. S. Fault Diagnosis in Rotating Machines Using Vibration Monitoring and Artificial Neural Network. Ph.D. Thesis, Delhi, 2002.

[4] Pandey S., Nakra B. C. Vibration Monitoring of a rotor system using RMS acceleration. International Journal of Engineering Science and Technology, Vol. 3, Issue 4, 2011, p. 2559-2572.

[5] Vyas N. S., Satish Kumar D. Artificial neural network design for the fault identification in a rotor bearing system. Mechanism and Machine Theory, Vol. 36, 2001, p. 157-175.

[6] Technotes. Spectra Quest Inc., 2012.

[7] Eisenmann R. C. Machinery Malfunction Diagnosis and Correction: Vibration Analysis and Troubleshooting for the Process Industries. Prentice Hall, 1997.

[8] Li Eric, Ganeriwala Suri Vibration and Force Signatures of Overhung Rotor Rotating Machine. Technote, Spectra Quest Inc., 2008.

[9] Kelm R. D., Kelm P. E. Advanced field balancing techniques. Vibration Institute Annual Training Seminar, 2008. 